\title{
Comparison of mesiodistal root angulation with posttreatment panoramic radiographs and cone-beam computed tomography
}

\author{
Daniel G. Bouwens ${ }^{a}$ [Resident], Lucia Cevidanes ${ }^{b}$ [Assistant professor], John B. \\ Ludlow ${ }^{\mathrm{C}}$ [Professor], and Ceib Phillips ${ }^{\mathrm{d}}$ [Professor] \\ aDepartment of Orthodontics School of Dentistry, University of North Carolina, Chapel Hill \\ ${ }^{b}$ Department of Orthodontics School of Dentistry, University of North Carolina, Chapel Hill \\ 'Department of Diagnostic Sciences \& General Dentistry School of Dentistry, University of North \\ Carolina, Chapel Hill \\ ${ }^{\mathrm{d} D e p a r t m e n t}$ of Orthodontics School of Dentistry, University of North Carolina, Chapel Hill
}

\begin{abstract}
Introduction-Orthodontists assess mesiodistal root angulations before, during, and after orthodontic treatment as an aid in establishing proper root position. Panoramic imaging has been useful for this purpose and is a valuable screening tool in diagnosis and planning treatment of orthodontic patients. Cone-beam computed tomography (CBCT) for imaging of the craniofacial complex creates the opportunity to evaluate 3 -dimensional images compared with traditional 2dimensional images. The purpose of this project was to compare mesiodistal root angulations by using posttreatment panoramic radiographic images and CBCT scans.
\end{abstract}

\begin{abstract}
Methods-Mesiodistal root angulations from panoramic images and CBCT scans of 35 orthognathic surgery patients after orthodontic treatment were compared. The panoramic images were measured by using VixWin (Gendex Dental Systems, Des Plaines, Ill), and the CBCT scans by using InvivoDental 3D (version 4.1, Anatomage, San Jose, Calif). The mesiodistal root angulation of each maxillary and mandibular tooth was measured by using the occlusal plane as the reference line. With an intercept-only linear regression for correlated data (with an unstructured covariance structure), the global test of whether the mean vector of all differences for the teeth is zero was performed separately for the 2 arches.
\end{abstract}

Results-The global test for both arches was statistically significant $(P<0.001)$, indicating an overall difference in root angulation between measurements from panoramic and $\mathrm{CBCT}$ images. There was no discernible pattern in the average differences between panoramic and $\mathrm{CBCT}$ measurements.

Conclusions-The assessment of mesiodistal tooth angulation with panoramic radiography should be approached with caution and reinforced by a thorough clinical examination of the dentition.

During orthodontic treatment, the alignment of the roots of the teeth in parallel axial inclinations is critical for the correct alignment and occlusion of the teeth and for maintaining a stable orthodontic result. Panoramic radiographs have traditionally been used

Copyright $\odot 2011$ by the American Association of Orthodontists

Reprint requests to: Ceib Phillips, University of North Carolina, School of Dentistry, Campus Box 7450, Chapel Hill, NC 72599-7450; ceib_phillips@dentistry.unc.edu..

The authors report no commercial, proprietary, or financial interest in the products or companies described in this article. 
as a diagnostic aid before, during, and after orthodontic treatment to assess root position. The clinical examination portion of the American Board of Orthodontics certification requires the submission of panoramic radiographs for documentation of root inclination and parallelism after treatment. ${ }^{1}$

Unfortunately, because panoramic image generation necessitates large beam deviations from the perpendicular to the object and film, tooth position and inclination can be distorted or magnified by varying amounts during acquisition. Numerous investigators have evaluated angular distortion in panoramic images, especially with regard to tooth inclination. These investigations have demonstrated that panoramic images have limits when used for the assessment of mesiodistal angulations. ${ }^{2-8}$ Angular distortion in the radiograph results from the combined variable distortions in the horizontal and vertical dimensions. ${ }^{8}$ The angulations measured from panoramic images relative to known angulations have demonstrated significant alterations in the mesiodistal angulations for most maxillary and mandibular teeth. ${ }^{2-5,9}$

Cone-beam computed tomography (CBCT) of the craniofacial complex provides the opportunity to evaluate patient anatomy, including the occlusion and tooth angulations, in 3 dimensions. Peck et $\mathrm{al}^{10}$ used CBCT for comparison with panoramic images before orthodontic treatment in 5 subjects. The angulations measured from the panoramic images were different from those based on CBCT reconstructed panoramic images, and the standard panoramic images produced a false impression of mesial tilt on the maxillary anterior teeth and distal tilt on the maxillary posterior teeth, but there was no recognizable pattern in the mandible. The conclusion was that the values from CBCT reconstructed panoramic images were more accurate because CBCT reconstructions do not have the distortions inherent in 2dimensional panoramic radiograph acquisitions. The purpose of this project was to compare the mesiodistal root angulations measured from posttreatment panoramic radiographic images and CBCT volumes, rather than from reconstructed panoramic images. Study participants had completed orthodontic treatment and orthognathic surgery and received concurrent panoramic radiographs and NewTom CBCT (3G, AFP Imaging, Elmsford, NY) scans after the postsurgical orthodontic phase of treatment (approximately 1 year after surgery).

\section{MATERIAL AND METHODS}

Study subjects were obtained from a review of the clinical records of 155 subjects who had consented to participate in an ongoing prospective observational project (NIH grant DE 05215, "influences on stability following orthognathic surgery"), approved by the Biomedical Institutional Review Board, between July 21, 2003, and May 1, 2008. Each subject signed a consent form (assent with parental permission) and a HIPAA consent for the use of clinical records. The inclusion criteria were concurrent panoramic and CBCT images taken after the postsurgical phase of orthodontic treatment (approximately 1 year after surgery) and a panoramic radiograph obtained by using the Orthophos XG Plus (Sirona Dental Systems, Charlotte, NC). Subjects were excluded if they had at least 1 dilacerated root per quadrant because of the difficulty in determining the long axis of the tooth, or if more than 1 tooth per quadrant anterior to the first molar was missing.

Each subject was assigned a random identification number so that the examiner (D.G.B.) would be masked to the subject when measuring the panoramic radiographs and the CBCT scans. Panoramic images were captured via a charge-coupled device image sensor and imported to the VixWin (Gendex Dental Systems, Des Plaines, Ill) software package for measurement (Fig 1). CBCT scans were obtained in DICOM (digital imaging and communications in medicine) format by using the NewTom $3 \mathrm{G}$ and were accessed by using 
InvivoDental 3D (version 4.1, Anatomage, San Jose, Calif) imaging software to allow measurements (Fig 2).

Mesiodistal root angulations were measured from the first molar anteriorly for all teeth. All measurements were made by using the occlusal plane as the reference line. The occlusal plane was constructed in the panoramic image by connecting the cusp tips of all teeth and in the CBCT volume by orienting the occlusal plane parallel to the lower border of the display window from the sagittal and coronal views. The long axis of the tooth was determined to complete each angular measurement relative to the occlusal plane. In the CBCT volume and panoramic radiograph, the long axis of the tooth was defined by the buccal cusp tip or midpoint of the incisal edge and the root apex for single-rooted teeth, and the occlusal aspect of the buccal groove and the depth of the bifurcation or trifurcation for multi-rooted teeth. In the $\mathrm{CBCT}$ volume, custom sections were created from the axial slice, and each measurement was made from a facial view of the tooth in the posterior. For the CBCT measurements of the anterior teeth, the volume rendering was reoriented to view the tooth from the facial aspect, and a custom section was created from the sagittal slice before measurement of the tooth.

Ten subjects were randomly selected. The measurement procedures (importing the images and making measurements) were repeated with 1 week between the initial and the replicate measurements. Reliability and systematic bias were assessed separately for the panoramic and $\mathrm{CBCT}$ images by using intraclass correlation statistics and paired t tests, respectively.

\section{Statistical analysis}

To assess the differences in mesiodistal root angulations between the panoramic images and the CBCT scans, an intercept-only linear regression for correlated data, accounting for the correlation in each subject, with an unstructured covariance was fit separately for the maxillary and mandibular arches. These models examined whether the differences between the CBCT scans and the panoramic images were nonzero, and, if so, whether the differences were related to tooth location.

\section{RESULTS}

The clinical records of 155 subjects who had consented to participate in an ongoing prospective observational project between July 21, 2003, and May 1, 2008, were reviewed; 118 subjects were imaged with a panoramic machine other than the Orthophos XG Plus, and 2 subjects were excluded due to several missing teeth or dilacerated roots in 1 quadrant, resulting in 35 subjects in the sample. Approximately $54 \%$ of the subjects were female, and $83 \%$ were white, with an average age of $21.2 \pm 6.5$ years.

The intraclass correlation coefficients ranged from 0.98 to 1.0 for the measurements obtained from the panoramic radiographs and from 0.99 to 1.0 for the CBCT measurements, indicating excellent intraobserver reliability. No mean difference for the replicate CBCT measurements was statistically significant $(P>0.05)$. The mean differences for the replicate panoramic angulations for maxillary right canine and first molar and left lateral incisor were statistically significantly different $(P<0.05)$ from zero; the replicate measurements tended to be slightly greater than the first set; however, no mean difference was greater than $0.5^{\circ}$.

The mean differences between the panoramic and the CBCT angulations for both arches were statistically significant $(P<0.0001)$. There was no clear pattern for the differences in either arch (Tables I and II), although the differences between the panoramic and CBCT angulations were statistically significantly different for all maxillary anterior teeth. There 
were statistically significant differences in mesiodistal tooth angulations for $75 \%$ of maxillary and $67 \%$ of mandibular teeth.

Prior investigations indicated that variations up to $5^{\circ}$ in mesiodistal tooth angulation relative to an established reference plane do not alter treatment decisions during the assessment of tooth angulation on a panoramic radiograph. ${ }^{5,7,11,12}$ Application of this clinically significant tolerance limit indicates that $34 \%$ of maxillary and $38 \%$ of mandibular image angles from panoramic radiographs were clinically significantly different from angles represented in the CBCT volumes when evaluated on a tooth-by-tooth basis.

\section{DISCUSSION}

Most orthodontists use midtreatment panoramic radiographs to evaluate axial tooth inclinations to either reposition the brackets or place detailing bends in the archwire to enhance axial tooth positions. An understanding of anticipated deviations in axial tooth positions represented by the panoramic radiograph is important clinically. Previous investigators found significant inaccuracies in mesiodistal tooth angulations in panoramic radiographs. They attributed the inaccuracy of panoramic images to projection geometry, focal trough depth and geometry, variable vertical and horizontal magnification factors, and patient positioning errors. ${ }^{2,5,13-17}$ Although there is potential for image distortion from a slight wobble during unit rotation in CBCT image acquisition, a correction algorithm in the software removes the distortion before image reconstruction. The accuracy of a CBCT volume is typically limited only by resolution or pixel size. ${ }^{18-20}$ Several studies have verified the accuracy of measurements from CBCT image volumes, with most focusing on linear measurements. ${ }^{18,20,21}$ Additionally, Marmulla et al ${ }^{19}$ concluded that the digital volume tomographies of the NewTom 9000 provide images that are geometrically correct, and Mischkowski et $\mathrm{al}^{22}$ concluded that the CBCT device (Galileos) provided acceptable information about linear distances and volumes. This study was undertaken to assess the deviation in axial inclination depicted by panoramic radiographs compared with CBCT volumes in a sample of patients after orthodontic treatment to identify panoramic errors that could be expected then.

The results of this investigation indicated that most panoramic image angulations were statistically significantly different from those represented in the CBCT volumes. For clinical purposes, it has been established by previous investigators that variations over $5^{\circ}$ between a tooth and an established reference plane create significant changes during the assessment of tooth angulations on a panoramic radiograph. ${ }^{5,7,11,12}$ Application of these clinically significant tolerance limits indicated that $43 \%$ of maxillary anterior, $24 \%$ of maxillary posterior, $39 \%$ of mandibular anterior, and $36 \%$ of mandibular posterior image angles from panoramic radiographs were clinically significantly different from the angles represented in the CBCT volumes. A more in-depth review of individual teeth in the anterior region showed clinically significant deviations for $43 \%$ of maxillary lateral incisors, $74 \%$ of maxillary canines, $49 \%$ of mandibular lateral incisors, and $30 \%$ of mandibular canines (Figs 3 and 4). These data support recent changes by the American Board of Orthodontics to exclude scoring of the canines when assessing root angulations via panoramic radiographs. However, the data also indicate that it would be judicious to exclude lateral incisor evaluations by panoramic radiographs.

The direction of the differences between mesiodistal tooth angulations on the panoramic radiographs and $\mathrm{CBCT}$ images when assessing all teeth, except the maxillary first molars, indicated that the following teeth had exaggerated mesial root tip on the panoramic radiograph: maxillary lateral incisors and canines, and mandibular premolars; the remaining teeth exhibited exaggerated distal root tip. However, the only area where statistically 
significant differences were observed on both sides of the dental arch was in the maxillary anterior region.

For all maxillary anterior teeth, the panoramic image demonstrated exaggerated distal root tip for the central incisors and exaggerated mesial root tip for the lateral incisors and canines. The exaggeration of mesial root tip for maxillary lateral incisors and canines agrees with previous investigations that recognized overinclination in the mesial direction for all maxillary anterior teeth from panoramic images. The findings regarding central incisor angulation were opposite to those reported by Mckee et $\mathrm{al}^{3}$ and Peck et al. ${ }^{10}$ Although statistically significant differences were not obtained bilaterally for the maxillary posterior teeth, the directionality of the differences was the same for the first and second premolars; both exhibited exaggerated distal root tip on the panoramic images relative to the CBCT volumes. This finding supports overstated root divergence between maxillary canines and first premolars as previously reported. $3,9,10$

For the mandible, the statistically significant differences between the 2 images on the right and left sides were not consistent. However, the directional pattern of the differences indicates exaggerated distal root tip of central incisors, lateral incisors, canines, and first molars, and exaggerated mesial root tip in the premolar region on the panoramic images relative to the CBCT volumes. These results contradict those of Mckee et al, ${ }^{3}$ who found exaggerated mesial inclination of the roots with the greatest discrepancy between the lateral incisors and the canines, where root parallelism was misrepresented as root convergence. We found the greatest discrepancy between the 2 images for the canines and first premolars, with exaggerated root convergence in this region on the panoramic images.

Panoramic radiographs are frequently used by clinicians during treatment to evaluate and adjust mesiodistal tooth angulations. The risk is that a change of inclination of the teeth in the buccolingual direction can appear as a change in mesiodistal tooth angulation. Increased lingual root torque frequently appears as more mesial root tip on the panoramic image, whereas increased buccal root torque results in more distal root tip. The effect of a buccolingual angulation on the mesiodistal angulation is inconsistent, and extensive variability has been reported. ${ }^{4,9}$

Although we used conventional slices and custom sections for the measurement of mesiodistal tooth inclination, the 3-dimensional (3D) renderings of CBCT volumes might provide a more powerful and simplified tool for the visualization of root angulation and proximity by the clinician rather than making assessments using conventional slices. However, the 3D renderings were not an effective tool for the measurement of mesiodistal tooth inclination because of the difficulty in accurately selecting points in the volume and localizing points for measurement in the same plane (Fig 5).

The method of image acquisition for this study potentially introduced increased variability in the panoramic radiographs measurements. Panoramic radiographs were obtained by trained technicians in the radiology clinic at the University of North Carolina as a part of an ongoing prospective observational project that also included a CBCT volume and a cephalometric radiograph. The panoramic radiographs were captured with 1 of 5 panoramic units housed in the radiology clinic, but only those obtained with the OrthoPhos XG Plus were included in this study. Other panoramic units were excluded because of the difficulty in ascertaining which images were acquired with particular units and the limited numbers of images attributable to specific panoramic units. Therefore, the results of this study relate only to the particular panoramic unit used. Mckee $3 \mathrm{et} \mathrm{al}^{3}$ found a similar pattern of deviations in mesiodistal tooth angulations compared with known angulations for 4 different units when panoramic radiographs were obtained in a standardized position. The 
investigators included a prior generation of the unit that we used (Orthophos) and reported results comparable with the other 3 units (OP100 [Instrumentarium, Tuusula, Finland], Cranex 31 [Orion Soredex, Helsinki, Finland], and PM 2002 EC Proline [Planmeca, Helsinki, Finland]).

The variability in personnel and the lack of a standard protocol for using a specific unit in the ongoing prospective observational project might have increased the likelihood of patient positioning errors, since the technicians were required to use several panoramic units and could have developed varying degrees of comfort with each unit. Additionally, the use of 1 panoramic unit that can only be approached from 1 direction to position the patient might result in systematic errors in positioning; however, this was unlikely, since the radiographs were not acquired by 1 technician. Previous investigations indicated great variability and significant differences in panoramic image axial tooth inclinations when patient positioning was varied. ${ }^{2,6,13,12}$ Ludlow et $\mathrm{a}^{18}$ indicated that patient-positioning difficulties associated with panoramic images are not present in CBCT images when assessing hard tissues.

However, it was suggested that soft-tissue assessment can be altered by patient positioning because some CBCT units require a supine rather than a seated position for image acquisition.

\section{CONCLUSIONS}

1. The panoramic radiograph remains a useful screening instrument for the evaluation of present and missing or supernumerary teeth, dental age, and eruption sequence. However, panoramic images provide less reliable information regarding mesiodistal tooth angulations and might exhibit deviations in both mesial and distal directions for all teeth.

2. When using panoramic radiographs for assessment of mesiodistal tooth angulations throughout treatment, the radiographic data must be combined with a thorough intraoral evaluation to produce the most satisfactory results.

3. CBCT allows clinicians to obtain $3 \mathrm{D}$ images of the craniofacial complex with similar absorbed doses as dental radiographs, and the 3D volume renderings provide a powerful tool for the visualization of root angulation.

\section{REFERENCES}

1. American Board of Orthodontics. Grading system for dental casts and panoramic radiographs. American Board of Orthodontics; St. Louis, MO: 2008. p. 17-8.

2. Mckee IW, Glover KE, Williamson PC, Lam EW, Heo G, Major PW. The effect of vertical and horizontal head positioning in panoramic radiography on mesiodistal tooth angulations. Angle Orthod 2001;71:442-51. [PubMed: 11771782]

3. Mckee IW, Williamson PC, Lam EW, Heo G, Glover KE, Major PW. The accuracy of 4 panoramic units in the projection of mesiodistal tooth angulations. Am J Orthod Dentofacial Orthop 2002;121:166-7. [PubMed: 11840131]

4. Lucchesi MV, Wood RE, Nortje CJ. Suitability of the panoramic radiograph for assessment of mesiodistal angulation of teeth in the buccal segments of the mandible. Am J Orthod Dentofacial Orthop 1988;94:303-10. [PubMed: 3177283]

5. Stramotas S, Geenty JP, Petocz P, Darendeliler MA. Accuracy of linear and angular measurements on panoramic radiographs taken at various positions in vitro. Eur J Orthod 2002;24:43-52. [PubMed: 11887378]

6. Philipp RG, Hurst RV. The cant of the occlusal plane and distortion in the panoramic radiograph. Angle Orthod 1978;48:317-23. [PubMed: 281889] 
7. Tronje G, Welander U, McDavid WD, Morris CR. Image distortion in rotational panoramic radiography. III. Inclined objects. Acta Radiol Diagn (Stockh) 1981;22:585-92. [PubMed: 7331872]

8. Wyatt DL, Farman AG, Orbell GM, Silveira AM, Scarfe WC. Accuracy of dimensional and angular measurements from panoramic and lateral oblique radiographs. Dentomaxillofac Radiol 1995;24:225-31. [PubMed: 9161166]

9. Samawi SS, Burke PH. Angular distortion in the orthopantomo-gram. Br J Orthod 1984;11:100-7. [PubMed: 6587906]

10. Peck JL, Sameshima GT, Miller A, Worth P, Hatcher DC. Mesiodistal root angulation using panoramic and cone beam CT. Angle Orthod 2007;77:206-13. [PubMed: 17319753]

11. Samfors KA, Welander U. Angle distortion in narrow beam rotation radiography. Acta Radiol Diagn (Stockh) 1974;15:570-6. [PubMed: 4451143]

12. Hardy TC, Suri L, Stark P. Influence of patient head positioning on measured axial tooth inclination in panoramic radiography. J Orthod 2009;36:103-10. [PubMed: 19487740]

13. McDavid WD, Tronje G, Welander U, Morris CR. Dimensional reproduction in rotational panoramic radiography. Oral Surg Oral Med Oral Pathol 1986;62:96-101. [PubMed: 3460015]

14. Xie Q, Soikkonen K, Wolf J, Mattila K, Gong M, Ainamo A. Effect of head positioning in panoramic radiography on vertical measurements: an in vitro study. Dentomaxillofac Radiol 1996;25:61-6. [PubMed: 9446974]

15. Mayoral G. Treatment results with light wires studied by panoramic radiography. Am J Orthod 1982;81:489-97. [PubMed: 6960734]

16. Scarfe WC, Nummikoski P, McDavid WD, Welander U, Tronje G. Radiographic interproximal angulations: implications for rotational panoramic radiography. Oral Surg Oral Med Oral Pathol 1993;76:664-72. [PubMed: 8247510]

17. Welander U, McDavid WD, Tronje G, Morris CR. An analysis of different planes within the image layer in rotational panoramic radiography. Dentomaxillofac Radiol 1987;16:79-84. [PubMed: 3507323]

18. Ludlow JB, Laster WS, See M, Bailey LJ, Hershey HG. Accuracy of measurements of mandibular anatomy in cone beam computed tomography images. Oral Surg Oral Med Oral Pathol Oral Radiol Endod 2007;103:534-42. [PubMed: 17395068]

19. Marmulla R, Wortche R, Muhling J, Hassfeld S. Geometric accuracy of the NewTom 9000 cone beam CT. Dentomaxillofac Radiol 2005;34:28-31. [PubMed: 15709102]

20. Lascala CA, Panella J, Marques MM. Analysis of the accuracy of linear measurements obtained by cone beam computed tomography (CBCT-NewTom). Dentomaxillofac Radiol 2004;33:291-4. [PubMed: 15585804]

21. Kobayashi K, Shimoda S, Nakagawa Y, Yamamoto A. Accuracy in measurement of distance using limited cone-beam computerized tomography. Int J Oral Maxillofac Implants 2004;19:228-31. [PubMed: 15101594]

22. Mischkowski RA, Pulsfort R, Ritter L, Neugebauer J, Brochhagen HG, Keeve E, et al. Geometric accuracy of a newly developed cone-beam device for maxillofacial imaging. Oral Surg Oral Med Oral Pathol Oral Radiol Endod 2007;104:551-9. [PubMed: 17613260] 


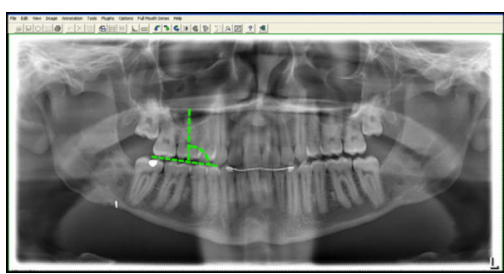

Fig 1.

Panoramic measurement with VixWin. 


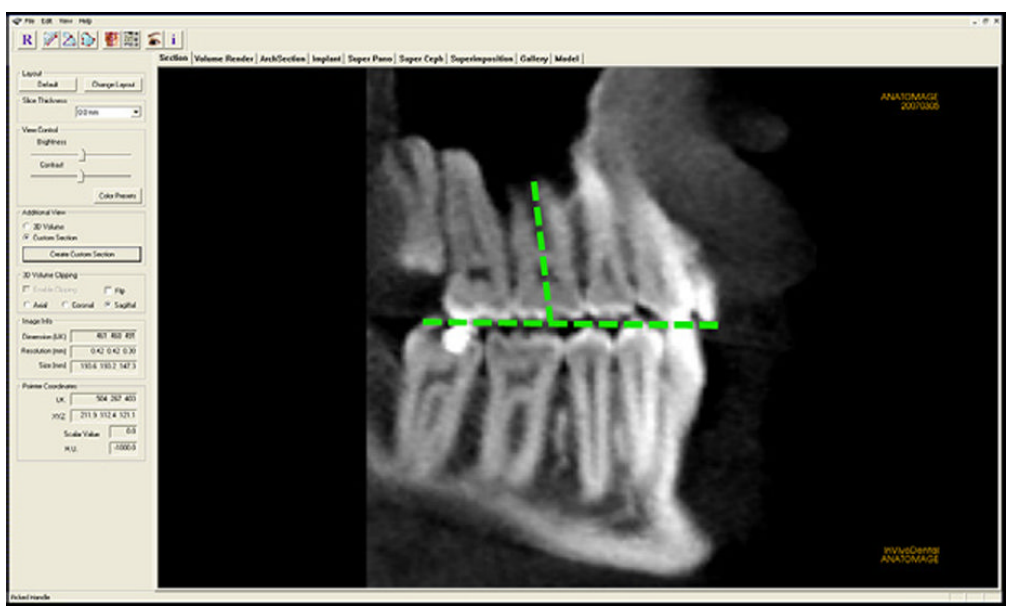

Fig 2.

CBCT measurement with InvivoDental. 


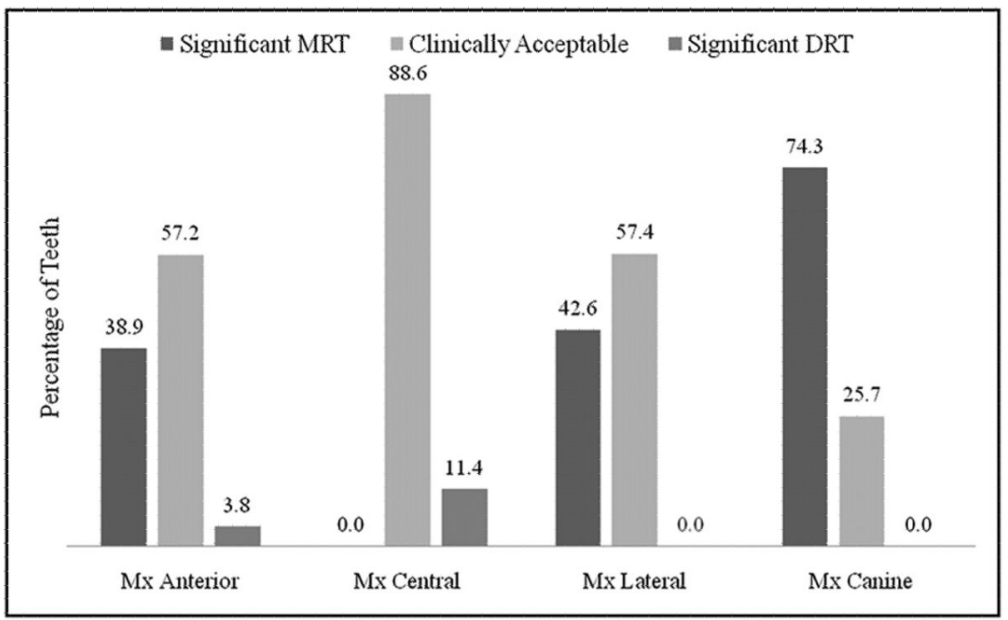

Fig 3.

Clinically significant mesial $(M R T)$ and distal $(D R T)$ deviations for the maxillary anterior teeth. 


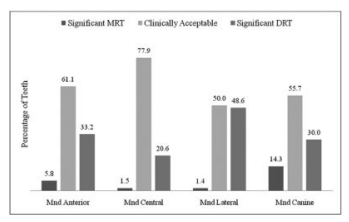

Fig 4.

Clinically significant mesial (MRT) and distal (DRT) deviations for the mandibular anterior teeth. 

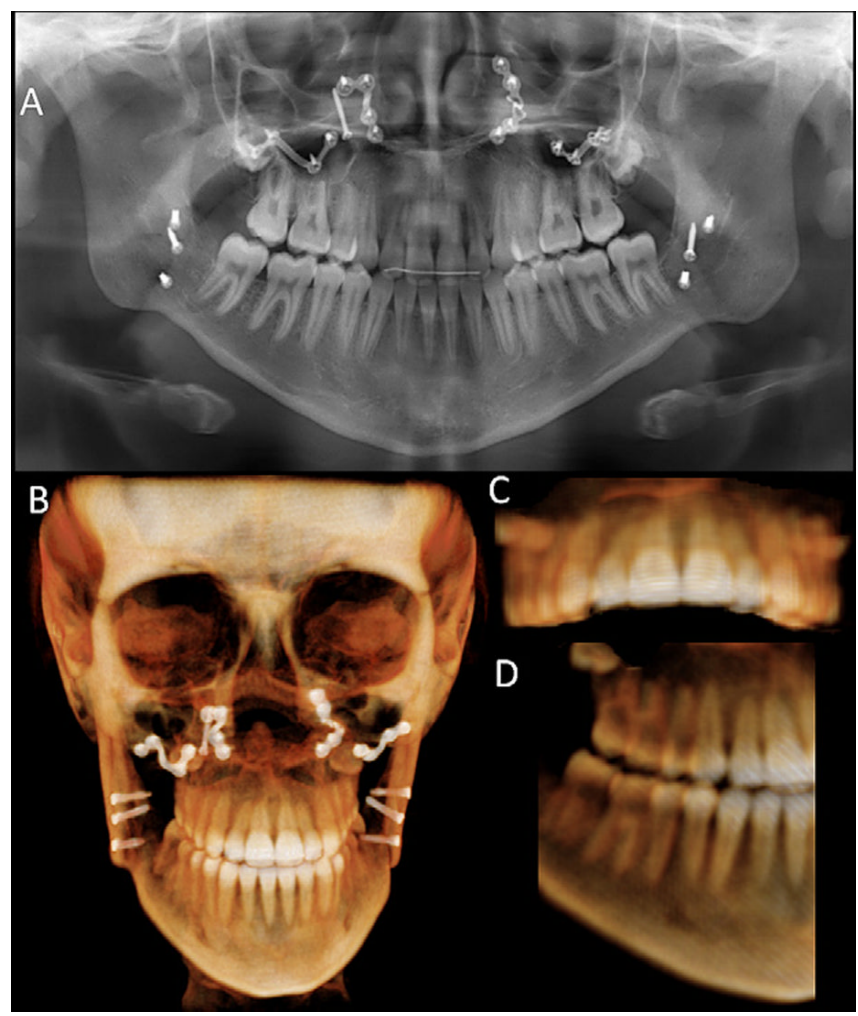

Fig 5.

A, Panoramic radiograph demonstrating excessive mesial root tip of a maxillary right lateral incisor; $\mathbf{B}$, frontal view of CBCT volume rendering also gives a perception of the root proximity between the maxillary right central and lateral incisors; $\mathbf{C}$, anterior view of the maxillary dentition demonstrates the torque differential between the right lateral incisor and the central incisor; $\mathbf{D}$, right three quarter view of the dentition shows the maxillary right lateral incisor adequately positioned relative to the central incisor. 


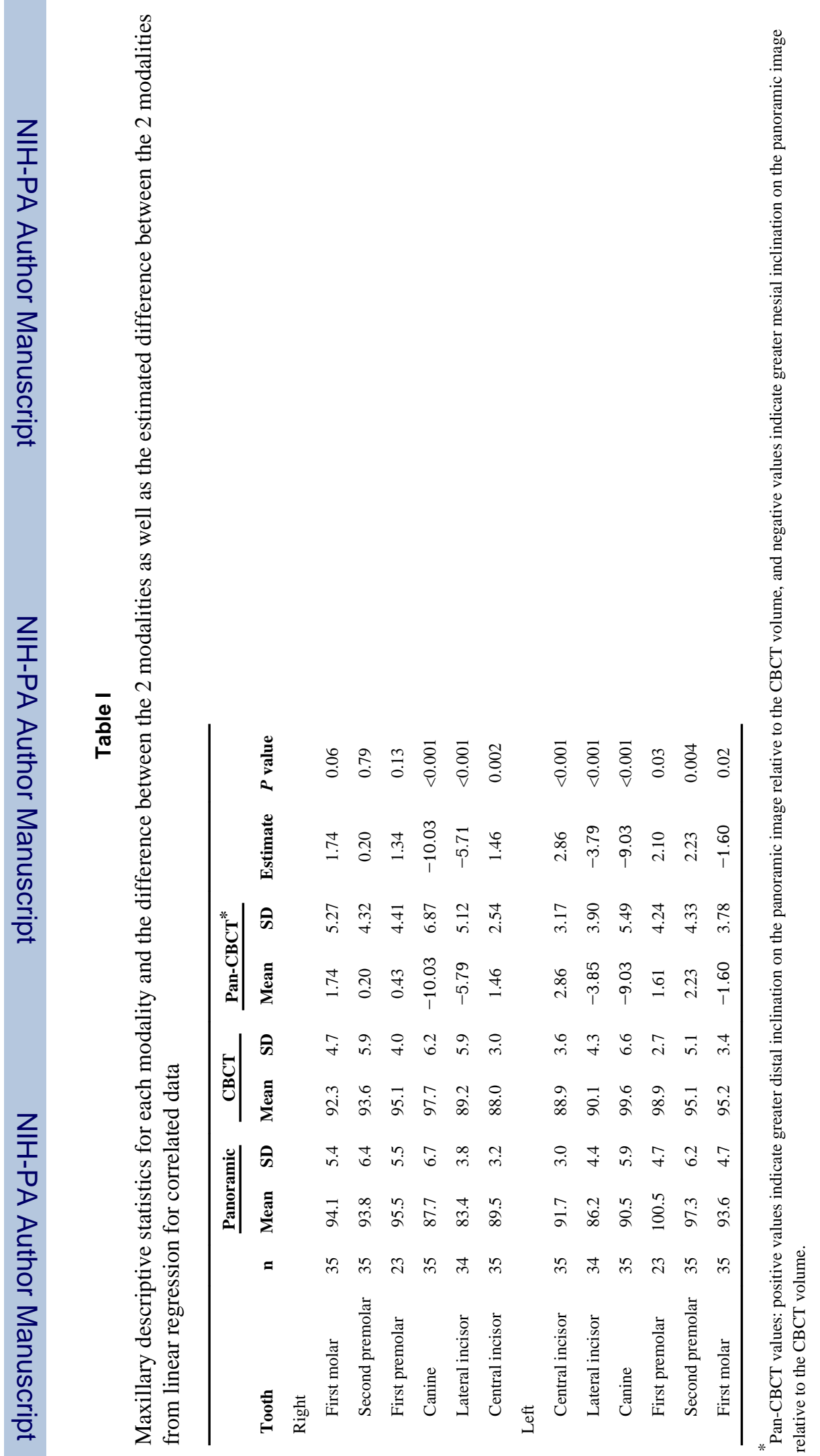




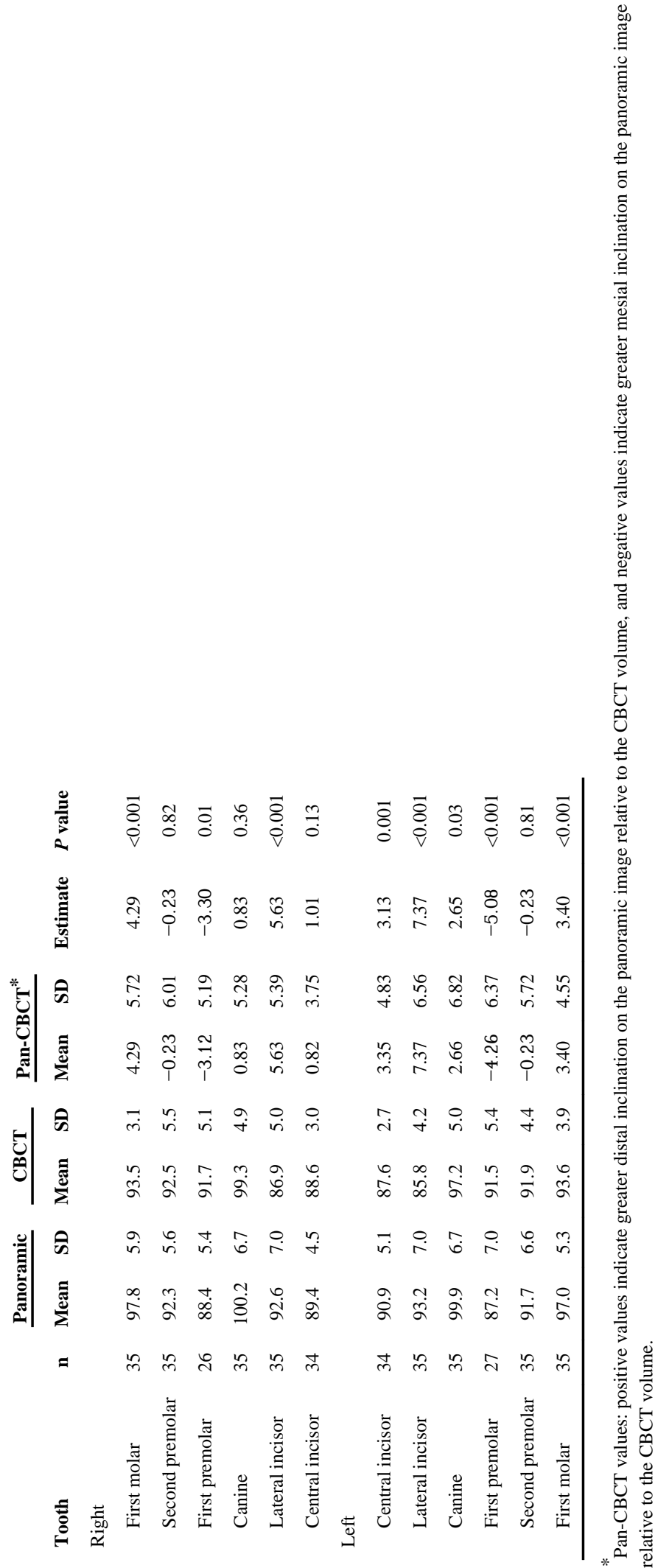

